\title{
Twin beams, nonlinearity, and walk-off in optical parametric oscillators
}

\author{
Roberta Zambrini and Maxi San Miguel \\ Instituto Mediterráneo de Estudios Avanzados, IMEDEA (CSIC-UIB), Campus Universitat Illes Balears, \\ E-07071 Palma de Mallorca, Spain
}

(Received 10 November 2001; published 8 August 2002)

\begin{abstract}
We study the quantum properties of the spatially tilted macroscopic signal beams in the transverse pattern formed in a degenerate optical parametric oscillator above threshold. We show that walk-off leads to an imbalance in the intensities and fluctuations of these beams when nonlinear multimode interactions are effective. Still, quantum correlations between the two beams are preserved, so that their intensity difference exhibits sub-Poissonian statistics.
\end{abstract}

DOI: 10.1103/PhysRevA.66.023807

PACS number(s): 42.50.Dv, 42.65.Sf, 42.50.Ct

Nonlinear wave-mixing processes are fundamental to produce nonclassical states of light. Specially interesting in this context is the process of parametric down-conversion (PDC), in which a pump photon at frequency $\omega_{0}$, interacting with a $\chi^{(2)}$ crystal, decays into two highly correlated twin signal photons at lower frequencies $\omega_{1}, \omega_{2}$. Nonclassical states obtained by PDC have found a wide number of applications [1]. The photon number difference between the downconverted fields in PDC, $\hat{N}_{D}=\hat{N}_{1}-\hat{N}_{2}$, is a constant of motion (Manley-Rowe relations [2]): at each time the pump creates a photon at $\omega_{1}$ it must also create a photon at $\omega_{2}$, leading to a nonclassical state with sub-Poissonian statistics in $\hat{N}_{D}$. Note that this result is also valid in lossy systems for the intracavity steady state averages $\left\langle\hat{N}_{D}\right\rangle,\left\langle\hat{N}_{D}^{2}\right\rangle$, as shown [3] in a master equation formalism.

Reynaud and co-workers [4] considered PDC inside an optical cavity and suggested the use of the nondegenerate optical parametric oscillator (NDOPO) in order to generate macroscopic nonclassical states instead of single photon pairs. Cavity effects introduce a threshold, yielding two intense laserlike twin beams, distinguished by their polarization or frequency. Nonclassical statistics have been measured, with a maximum of $88 \%$ of reduction of fluctuations in $\hat{N}_{D}^{2}$ below the shot noise limit [5].

Diffraction effects in cavities with a large Fresnel number, combined with nonlinearities, produce transverse patterns above the threshold for down-conversion of the DOPO [6,7]. The possible quantum properties of these macroscopic patterns have been recently addressed [8]. In the simplest theoretical description, a stripe pattern, caused by the interference of two spatially tilted signal beams with transverse wave vectors $\pm \vec{k}_{c}$, emerges. These two beams have equal photon numbers and are entangled, i.e., their intensity difference is sub-Poissonian [9].

Macroscopic entanglement between the signal beams of opposite transverse wave vector in a pattern forming DOPO has been predicted, neglecting walk-off effects, below $[10,16]$ and at threshold [11]. The analysis at the threshold is based on a three-mode approximation $[3,9]$ and the prediction of entanglement is linked to considerations of transverse momentum conservation. In this paper, we consider the question of entanglement between the two macroscopic tilted sig- nal beams in the intense pattern that emerges above the threshold. In this regime two important aspects have to be taken into account. First, nonlinearity causes the emergence of harmonics of the transverse mode $k_{c}$ selected at threshold, so that a three-mode approximation is no longer valid. Second, walk-off, originating in the crystal birefringence, breaks the symmetry between the spatial beams and destroys the equivalence between the beams of wave vectors $\pm \vec{k}_{c}$. Our continuous analysis, which avoids few-mode approximations, shows that, neglecting walk-off effects, beam entanglement is preserved, but it does not follow from momentum conservation. When walk-off is not negligible, we show that one beam is more intense and it fluctuates more than the opposite one. Unexpectedly, also in the presence of such imbalance between the "twin" beams, the entanglement in fluctuations remains unchanged in the domain of parameters explored.

We consider a DOPO in type-I phase matching. In anisotropic birefringent media, rays do not necessarily travel in a direction perpendicular to their wave fronts; as a consequence there is misalignment between the Poynting vectors of the ordinarily polarized pump and the extraordinarypolarized signal, so that the signal walks off the pump. A detailed derivation of the classical equations of OPO including diffraction and walk-off effects is presented in Ref. [12], while linear and nonlinear stability analyses are discussed in Refs. $[13,14]$. The introduction of walk-off effects changes the field dynamics in several aspects. First, the pattern travels in the walk-off direction. Moreover, the drift term associated with the walk-off allows for a new intermediate regime close to the threshold, the convective regime, in which the pattern is sustained by quantum noise, and not by the dynamics [13]. The description of the quantum properties of the DOPO in the convective regime is given in Ref. [15]. In this paper we focus on the consequences of the walk-off in the absolute regime where the pattern is dynamically sustained. To this end, and for pedagogical reasons, we consider mainly an infinitely extended system in the transverse direction (plane wave pump and flat mirrors) so that no convective regime exists.

The DOPO Hamiltonian, $\hat{H}=\hat{H}_{0}+\hat{H}_{\text {int }}$, in a mean-field approximation is given by $[15,16]$ 


$$
\begin{aligned}
\hat{H}_{0}= & \int d^{2} \vec{x} \sum_{j=0,1}\left[\hat{A}_{j}^{\dagger}(\vec{x})\left(\Delta_{j}-a_{j} \nabla^{2}\right) \hat{A}_{j}(\vec{x})\right] \\
& +i v \hat{A}_{1}^{\dagger}(\vec{x}) \partial_{x} \hat{A}_{1}(\vec{x})+E_{0}\left[\hat{A}_{0}^{\dagger}(\vec{x})-\hat{A}_{0}(\vec{x})\right], \quad \vec{x}=(x, y) .
\end{aligned}
$$

$\hat{H}_{0}$ describes free propagation of the signal $\left(\hat{A}_{1}\right.$, first harmonic) and pump ( $\hat{A}_{0}$, second harmonic) fields in the cavity and the interaction with the external real pump $E_{0} ; a_{j}$ are the diffraction strengths, $v$ is the walk-off parameter, and $\Delta_{j}$ are cavity detunings;

$$
\hat{H}_{i n t}=i \frac{g}{2} \int d^{2} \vec{x}\left[\hat{A}_{0}(\vec{x}) \hat{A}_{1}^{\dagger^{2}}(\vec{x})-\hat{A}_{0}^{\dagger}(\vec{x}) \hat{A}_{1}^{2}(\vec{x})\right]
$$

describes the interaction between the first and the second harmonic fields.

We first note that the invariance under continuous translations in the transverse plane, implies that the total transverse momentum $\hat{\vec{P}}_{\perp}=\int \vec{k}\left[\hat{N}_{0}(\vec{k})+\hat{N}_{1}(\vec{k})\right] d \vec{k}$ is conserved in the Hamiltonian evolution $\left(\left[\hat{H}, \hat{P}_{\perp}\right]=0\right)$ and, in the presence of cavity losses, it has a zero steady state average $\left(\left\langle\hat{\vec{P}}_{\perp}\right\rangle\right.$ $=0)$. The momentum conservation, which is at the root of entanglement properties of the spatial beams [9] at the threshold, is independent of the walk-off term. In addition, this term appears only in the free Hamiltonian, and does not seem to affect the creation processes of twin photons governed by $\hat{H}_{i n t}$. In order to clarify the role of the walk-off in the photon number properties of different spatial modes, we consider, in the following, different approximations that disclose when the combination of the walk-off and nonlinearity becomes crucial.

Common approximations to study $\chi^{(2)}$ processes use quadratic Hamiltonians $[11,16,17]$. This is, in particular, a suitable approximation to study the DOPO below the threshold $\left[E_{0}<E_{0}^{t h r}=\sqrt{\left(1+\Delta_{0}\right)}\right]$. Neglecting pump depletion leads to the interaction PDC Hamiltonian

$$
\hat{H}_{i n t}^{L}=i \frac{g}{2} A_{0} \int d^{2} \vec{k}\left[\hat{A}_{1}^{\dagger}(\vec{k}) \hat{A}_{1}^{\dagger}(-\vec{k})-\text { H.c. }\right],
$$

where $A_{0}$ is the homogeneous constant classical pump. The total transverse momentum in this approximation reduces to $\hat{\vec{P}}_{\perp}^{L}=\int \vec{k} \hat{N}_{1}(\vec{k}) d \vec{k}$, and considering the total quadratic Hamiltonian $\hat{H}^{L}$ we have that $\left[\hat{H}^{L}, \hat{\vec{P}}_{\perp}^{L}\right]=0$ [18]. In particular, the intensity difference between any two modes with opposite transverse wave vector, $\hat{N}_{D}=\hat{N}_{1}(\vec{k})-\hat{N}_{1}(-\vec{k})$, commutes with $\hat{H}^{L}$. Therefore the properties of the intensity difference of twin beams below the threshold are not modified by the consideration of the walk-off. This conclusion is consistent with the fact that in the undepleted pump approximation the walk-off term can be removed from the dynamical equations by a change of reference frame, since the signal field is not coupled to any other dynamical variable.

The assumption of an undepleted pump is not appropriate above the threshold. A suitable nonlinear approximation immediately above the threshold of signal generation takes into

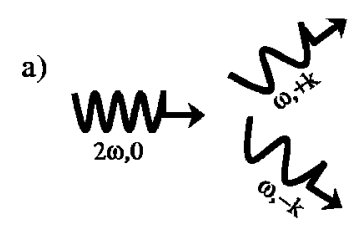

b)

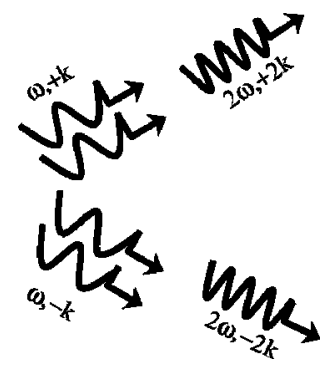

FIG. 1. (a) Three-mode interaction described by $\hat{H}_{\text {int }}^{(3)}$, Eq. (3). (b) Secondary processes: two subharmonic photons with the same transverse wave vector generate a photon with double frequency and wave vector.

account the dominant role of the three modes with operators $\hat{a}_{0,0}$ and $\hat{a}_{1, \pm \vec{k}_{c}}$ [19], where $\vec{k}_{c}$ is the transversal critical wave vector of the pattern $\left(\left|\vec{k}_{c}\right|^{2}=-\Delta_{1} / a_{1}[6]\right)$. The fundamental interaction [Fig. 1(a)] is the destruction of a photon of the homogeneous pump mode $\left(\vec{k}_{\perp}=\overrightarrow{0}\right)$ and the creation of two tilted signal twin photons with transverse wave vector $\pm \vec{k}_{c}$, described by the three-mode Hamiltonian

$$
\hat{H}_{i n t}^{(3)}=i g\left[\hat{a}_{0,0} \hat{a}_{1, \vec{k}_{c}^{\dagger}}^{\dagger} \hat{a}_{1,-\vec{k}_{c}}^{\dagger}-\text { H.c. }\right] .
$$

In this approximation and in a given spontaneously selected direction for the pattern, the total momentum is

$$
\hat{P}_{\perp}^{(3)}=k_{c}\left(\hat{a}_{1,+\vec{k}_{c}^{\dagger}} \hat{a}_{1,+\vec{k}_{c}}-\hat{a}_{1,-\vec{k}_{c}^{\dagger}} \hat{a}_{1,-\vec{k}_{c}}\right)=k_{c} \hat{N}_{D} .
$$

Momentum conservation $\left[\hat{H}^{(3)}, \hat{P}_{\perp}^{(3)}\right]=0$ implies that, independently of the walk-off, the twin beams have the same mean intensity and they are entangled [3,9]. Still, an effect of walk-off can be seen in the mean value of the Heisenberg equation

$$
\partial_{t}\left\langle\hat{a}_{1, \vec{k}_{c}}\right\rangle=\gamma\left(-1+i v k_{c}\right)\left\langle\hat{a}_{1, \vec{k}_{c}}\right\rangle+g\left\langle\hat{a}_{0,0} \hat{a}_{1,-\vec{k}_{c}}^{\dagger}\right\rangle .
$$

The walk-off term $i v k_{c}$ breaks the reflection symmetry (between $\vec{k}_{c}$ and $\left.-\vec{k}_{c}\right)$ resulting in a different phase evolution for $\left\langle\hat{a}_{1,+\vec{k}_{c}}\right\rangle$ and $\left\langle\hat{a}_{1,-\vec{k}_{c}}\right\rangle$.

This three-mode model gives only a good description of the DOPO extremely close to the threshold. Numerics indicate that increasing the pump intensity just $1 \%$ above the threshold there is significant feedback of the signal on the pump, and spatial harmonics come into play (multimode interaction). A nonlinear perturbative analysis of the process of pattern formation $[6,14]$ shows that, at first order in the distance to the threshold, the three-mode model is appropriate, but in second order also pump modes with transverse wave vector $\pm 2 \vec{k}_{c}$ are excited. A natural next approximation is then to take into account five modes in the interaction process: $\hat{a}_{0, \overrightarrow{0}}, \hat{a}_{0, \pm 2 \vec{k}_{c}}, \hat{a}_{1, \pm \vec{k}_{c}}$. The five-mode interaction Hamiltonian describes also the secondary processes of Fig. 1(b), namely, the combination of two tilted signal photons giving a pump photon with a double wave vector: 


$$
\hat{H}_{i n t}^{(5)}=\hat{H}_{i n t}^{(3)}+i \frac{g}{2}\left[\hat{a}_{0,2 \vec{k}_{c}} \hat{a}_{1, \vec{k}_{c}}^{\dagger+2}+\hat{a}_{0,-2 \vec{k}_{c}} \hat{a}_{1,-\vec{k}_{c}}^{\dagger 2}-\text { H.c. }\right] .
$$

The conserved transverse momentum is

$$
\hat{P}_{\perp}^{(5)}=k_{c}\left(\hat{N}_{1, \vec{k}_{c}}-\hat{N}_{1,-\vec{k}_{c}}\right)+2 k_{c}\left(\hat{N}_{0,2 \vec{k}_{c}}-\hat{N}_{0,-2 \vec{k}_{c}}\right) .
$$

The conservation law $\left[\hat{H}^{(5)}, \hat{P}_{\perp}^{(5)}\right]=0$ only imposes the restriction that the intensity difference $\hat{N}_{D}$ in the two signal beams is minus twice the intensity difference of the two pump tilted beams. But $\hat{N}_{D}$ is no longer a constant of motion and the commutator

$$
\left[\hat{H}^{(5)}, \hat{N}_{D}\right]=i g\left[-\hat{a}_{0,2 \vec{k}_{c}} \hat{a}_{1, \vec{k}_{c}}^{\dagger 2}+\hat{a}_{0,-2 \vec{k}_{c}} \hat{a}_{1,-\vec{k}_{c}}^{\dagger 2}-\text { H.c. }\right]
$$

is not fixed to take a zero value. Therefore, when a multimode nonlinear interaction is at work, the properties of the spatial beams $\hat{N}_{1}\left( \pm \vec{k}_{c}\right)$ might change: The photon number of a signal beam $\vec{k}_{c}$ might be larger than the photon number of the beam $-\vec{k}_{c}$, the difference compensating the difference in the photon number of the two pump tilted beams. This should not occur when there is no walk-off, because of symmetry considerations. However, walk-off implies the breakdown of reflection symmetry, which suggests having $\left\langle\hat{N}_{D}\right\rangle$ $\neq 0$ as a natural possibility. In fact, note that $\left[\hat{H}^{(5)}, \hat{N}_{D}\right]$ is phase sensitive and therefore dynamically modified by symmetry breaking caused by the walk-off [see Eq. (5)].

In order to substantiate the above ideas, we investigate numerically a continuous nonlinear model without constraint in the number of coupled modes. We consider a timedependent parametric approximation (TDPA) to the quantum formulation of the DOPO dynamics [15]. The approximation consists in taking the pump as a classical time-dependent $\mathcal{A}_{0}$ field. It is based on the existence of a macroscopic stable pump, both below and above the DOPO threshold. The onedimensional Langevin equations associated with the Wigner quasiprobability distribution in this formulation are [15]

$$
\begin{gathered}
\partial_{t} \mathcal{A}_{0}=\left[-\left(1+i \Delta_{0}\right)+i \partial_{x}^{2}\right] \mathcal{A}_{0}-\frac{1}{2} \alpha_{1}^{2}+E_{0}, \\
\partial_{t} \alpha_{1}=\left[-\left(1+i \Delta_{1}\right)+2 i \partial_{x}^{2}+v \partial_{x}\right] \alpha_{1}+\mathcal{A}_{0} \alpha_{1}^{*}+\xi(x, t),
\end{gathered}
$$

where space is scaled with the pump diffraction strength $a_{0}$, time is scaled with the cavity decay rate $\gamma$, and $\xi$ is a complex Gaussian white noise with nonvanishing correlation $\left\langle\xi(x, t) \xi^{*}\left(x^{\prime}, t^{\prime}\right)\right\rangle=1 / \sqrt{a_{0}}\left(g^{2} / \gamma^{2}\right) \delta\left(x-x^{\prime}\right) \delta\left(t-t^{\prime}\right)$.

Stochastic averages associated with $\alpha_{1}$ give the symmetrically ordered averages of corresponding operators in the signal field, driven by the "classical" pump field $\mathcal{A}_{0}$. This TDPA captures three fundamental features: first the nonlinearity of the system, i.e., the depletion of the pump (term $\alpha_{1}^{2}$ ); second the dynamical coupling between pump and signal, which, in particular, does not allow the elimination of the relative walk-off term changing the reference system; and third, the spatial multimode interaction allowing for the manifestation of symmetry breaking in the beam intensities.

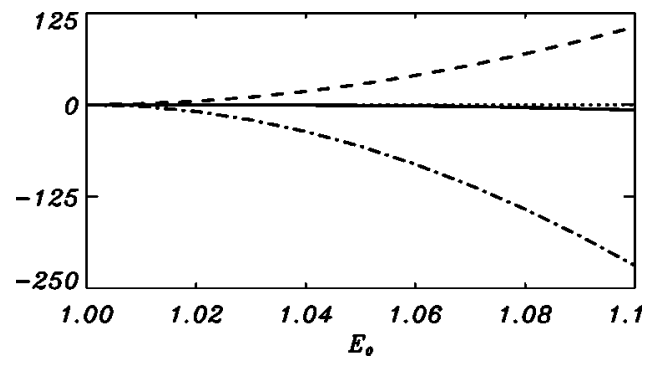

FIG. 2. $N_{1}\left(k_{c}\right)-N_{1}\left(-k_{c}\right)$ (dash-dotted line), $N_{0}\left(2 k_{c}\right)$ $-N_{0}\left(-2 k_{c}\right)$ (dashed line), and $P_{\perp}^{(5)} / k_{c}$ (continuous line) shown as a function of the pump $E_{0}$. Parameters: $v=0.42$, system size $L$ $=512 \Delta x=10\left(2 \pi / k_{c}\right), \Delta_{0}=0, \Delta_{1}=-0.25$. Pump threshold value: $E_{0}^{t h r}=1$. The dotted line is the zero value of $N_{1}\left(k_{c}\right)-N_{1}\left(-k_{c}\right)$ for $v=0$.

We first consider the nonlinear classical equations of the DOPO, given by Eq. (9) neglecting the noise source. When $v=0$, a stationary pattern appears and in the far field $N_{1}\left(k_{c}\right)=N_{1}\left(-k_{c}\right)$, even if-as shown above-this does not follow from a conservation law when there is a multimode interaction. On the other hand, when the walk-off is taken into account $(v \neq 0)$, broken symmetry leads to a tilted signal beam more intense than the signal beam of opposite transverse wave vector, in an amount that compensates the difference between the two tilted pump beams: In Fig. 2 we show that $N_{1}\left(k_{c}\right)-N_{1}\left(-k_{c}\right)=2\left[N_{0}\left(-2 k_{c}\right)+N_{0}\left(2 k_{c}\right)\right]$, so that the conservation law $P_{\perp}^{(5)}=0$ is fulfilled. However, this is only strictly true close enough to the threshold, where the five-mode approximation is valid. Already for the pump values shown in Fig. 2, we observe that $P_{\perp}^{(5)} \neq 0$. This indicates that a larger number of transverse modes are being excited and contribute to the conserved total transverse momentum $P_{\perp}$.

A microscopic interpretation of our findings in Fig. 2 is as follows: At threshold there is a primary process of annihilation of a pump photon creating two tilted signal photons (three-mode model), generating two beams such that $\left\langle\hat{N}_{1}\right.$ $\left.\left(+k_{c}\right)\right\rangle=\left\langle\hat{N}_{1}\left(-k_{c}\right)\right\rangle$. The secondary processes of Fig. 1(b) can happen with a different rate depending on the walk-off direction. Then, multimode interaction combined with broken reflection symmetry leads to a stronger depletion of one of the two signal beams.

A natural question is then whether quantum correlations are partially destroyed by the asymmetric depletion of the signal beams at $k_{c}$, as it happens in NDOPO (neglecting spatial dependence) when signal and idler have different losses [20]. To characterize such correlations we calculate from Eq. (9) the normal ordered variance normalized to the shot noise $\left(\mathcal{N}_{S}\right)$ value

$$
\mathcal{V}=\frac{\left\langle:\left[\hat{N}_{1}\left(k_{c}\right)-\hat{N}_{1}\left(-k_{c}\right)\right]^{2}:\right\rangle-\left\langle\hat{N}_{1}\left(k_{c}\right)-\hat{N}_{1}\left(-k_{c}\right)\right\rangle^{2}}{\mathcal{N}_{S}}
$$

Negative values of $\mathcal{V}$ indicate a nonclassical sub-Poissonian statistics for the intensity difference of the two signal beams at $\pm k_{c}$. Being $\mathcal{V}$ a normal ordered quantity, it is proportional 
to the output variance [22], indicating possible nonclassical signatures in the output fields correlations [23].

For the DOPO below the threshold, we find that the two opposite beams have the same variances (above the level of coherent states) and $\mathcal{V}=-0.5$ for $v=0$ and $v \neq 0$. This is in agreement with analytical calculations for a continuous linear model, which give-independently of the walk-off-a reduction of $50 \%$ in the fluctuations of the intensity difference with respect to the level of the coherent states inside the cavity [16]. Also at the threshold-where linearization fails due to undamped critical fluctuations, but a three-mode model is suitable-we obtain the same results, in agreement with Ref. [3]. Above the threshold, and if $v=0$, we still obtain $\left\langle\hat{N}_{D}\right\rangle=0$ and sub-Poissonian statistics with $\mathcal{V}=-0.5$. As discussed previously, given the multimode interaction, this result does not follow from momentum conservation alone. In fact, it is associated with the presence of a macroscopic spatial structure that dictates the spatial structure, symmetry properties, and correlations of the fluctuations [15].

Considering the effect of walk-off above the threshold $(v \neq 0)$ we find that the variances of the two beams become different, the most intense beam fluctuating more strongly. We obtain [21] $\left\langle\delta N_{1}\left(-k_{c}\right)^{2}\right\rangle /\left\langle\delta N_{1}\left(k_{c}\right)^{2}\right\rangle \simeq 1.005,1.01$, $1.02,1.03,1.04$ for pump values $E_{0} / E_{0}^{\text {thr }}$ $=1.01,1.03,1.05,1.07,1.1$, respectively. However, the correlation between these two nonequivalent beams is strong and macroscopic entanglement is preserved. In fact, within our accuracy, we get always the same value $\mathcal{V} \simeq-0.5$ obtained for $v=0$. We finally point out that the different noise levels of the two beams, as caused by the walk-off, is a general phenomenon that might explain experimental observations in other systems [24].

Our discussion so far has considered the ideal situation of a plane wave pump, but we have checked that our results are meaningful for a more physical spatial dependent wide pump profile. In this case the tilted beams have to be detected in a broad area of the size of the far field peaks [10]. We have performed numerical simulations in the absolute regime of a DOPO with a broad pump $E_{0}(x)=E_{t o p} E_{0}^{t h r} \exp$ $-\frac{1}{2}(x / 317)^{10}$ and $E_{\text {top }}=1.1$ [25]. In this situation we obtain $\mathcal{V}=3$ for $k=k_{c}$ if $\Delta k=2 \pi / L$; increasing the integration area around $k_{c}$ we find that $\mathcal{V}=-0.35$ for $3 \Delta k, \mathcal{V}=-0.40$ for $5 \Delta k$, and $\mathcal{V}=-0.49$ for $7 \Delta k$.

In the convective regime $\left(E_{t o p}=1.025\right)$ at $k=k_{c}$ we obtain $\mathcal{V} \simeq 10^{2}$, and integrating over a broad area of $30 \Delta k$ the variance decreases an order of magnitude, remaining always classical. In this regime the macroscopic entanglement is destroyed [15].

In conclusion, we predict quantum entanglement between the spatially tilted macroscopic signal beams of a DOPO above a threshold when the nonlinear multimode interaction is effective. The walk-off breaks the symmetry between the two beams, the more intense one becoming more noisy, but quantum entanglement is preserved.

We acknowledge financial support from the European Commission project QUANTIM (Grant No. IST-200026019) and the Spanish MCyT Project No. BFM2000-1108. Helpful discussions with D. Gomila, P. Colet, S. M. Barnett, and $\mathrm{M}$. Taki are also acknowledged.
[1] See, for example, references in A. Lamas-Linares, J.C. Howell, and D. Bowmeester, Nature (London) 412, 887 (2001); M. Atatüre, A.V. Sergienko, B.E.A. Saleh, and M.C. Teich, Phys. Rev. Lett. 86, 4013 (2001); The Physics of Quantum Information, edited by D. Bouwmeester, A. Ekert, and A. Zeilinger (Springer, Berlin, 2000).

[2] W.H. Louisell, A. Yariv, and A.E. Siegman, Phys. Rev. 124, 1646 (1961).

[3] R. Graham, Phys. Rev. Lett. 52, 117 (1984).

[4] S. Reynaud, Europhys. Lett. 4, 427 (1987); S. Reynaud, C. Fabre, and E. Giacobino, J. Opt. Soc. Am. B 4, 1520 (1987).

[5] J.R. Gao, F. Cui, C. Xue, C. Xie, and K. Peng, Opt. Lett. 23, 870 (1998).

[6] G-L. Oppo, M. Brambilla, and L.A. Lugiato, Phys. Rev. A 49, 2028 (1994).

[7] M. Vaupel, A. Maître, and C. Fabre, Phys. Rev. Lett. 83, 5278 (1999); S. Ducci, N. Trêps, A. Maitre, and C. Fabre, Phys. Rev. A 64, 023803 (2001).

[8] L.A. Lugiato, M. Brambilla, and A. Gatti, Adv. At., Mol., Opt. Phys. 40, 229 (1998).

[9] L.A. Lugiato and F. Castelli, Phys. Rev. Lett. 8, 3284 (1992); G. Grynberg and L.A. Lugiato, Opt. Commun. 101, 69 (1993).

[10] I. Marzoli, A. Gatti, and L.A. Lugiato, Phys. Rev. Lett. 78, 2092 (1997).

[11] F. Castelli and L.A. Lugiato, J. Mod. Opt. 44, 765 (1997).
[12] H. Ward, M.N. Ouarzazi, M. Taki, and P. Glorieux, Eur. Phys. J. D 3, 275 (1998), and references therein; The model we consider in this paper is derived under the standard conditions of slowly varying envelopes, mean field, single longitudinal mode, and triply resonant fields. For a discussion of the validity of the mean-field approximation see G-L. Oppo, A.J. Scroggie, and W.J. Firth, Phys. Rev. E 63, 066209 (2001); M. Tlidi, M. Le Berre, A. Ressayre, A. Tallet, and L. Di Menza, Phys. Rev. A 61, 043806 (2000); M. Le Berre, D. Leduc, S. Patrascu, E. Ressayre, and A. Tallet, Chaos, Solitons Fractals 10, 627 (1999).

[13] M. Santagiustina, P. Colet, M. San Miguel, and D. Walgraef, Phys. Rev. E 58, 3843 (1998).

[14] H. Ward, M.N. Ouarzazi, M. Taki, and P. Glorieux, Phys. Rev. E 63, 016604 (2000).

[15] R. Zambrini, S.M. Barnett, P. Colet, and M. San Miguel, Phys. Rev. A 65, 023813 (2002).

[16] A. Gatti, H. Wiedemann, L.A. Lugiato, I. Marzoli, G.L. Oppo, and S.M. Barnett, Phys. Rev. A 56, 877 (1997).

[17] R. Zambrini, M. Hoyuelos, A. Gatti, P. Colet, L. Lugiato, and M. San Miguel, Phys. Rev. A 62, 063801 (2000).

[18] In the rest of the paper the Hamiltonian without subscript means the sum of the corresponding $\hat{H}_{0}$ and the interaction term $\hat{H}_{i n t}$ in the different approximations considered. In any of 
these approximations $\left[\hat{H}_{0}, \hat{N}_{i}(k)\right]=0, i=0,1$.

[19] The operators $\hat{a}_{i, \vec{k}}(i=0,1)$ correspond to a discrete set of wave vectors and differ from $\hat{A}_{i}(\vec{k})$ in normalization factors.

[20] A.S. Lane, M.D. Reid, and D.F. Walls, Phys. Rev. A 38, 788 (1988); L. Lugiato and G. Grynberg, Europhys. Lett. 29, 675 (1995).

[21] We take $L=30\left(2 \pi / k_{c}\right)$ and other parameters as in Fig. 2.

[22] M.J. Collett and C.W. Gardiner, Phys. Rev. A 30, 1386 (1984).

[23] Outside the cavity, the quantity showing stronger quantum sig- natures is the spectral variance $V(\omega)$ [Eq. (19) in Ref. [10]]. The macroscopic entanglement, or total noise suppression, is reached at $\omega=0$. The integral over all frequencies of $V(\omega)$ corresponds to the equal times variance, proportional to the intracavity quantity $\mathcal{V}$ [Eq. (10)] calculated here.

[24] J.E. Sharping, M. Fiorentino, and P. Kumar, Opt. Lett. 26, 367 (2001).

[25] We take $L=51\left(2 \pi / k_{c}\right)$ and other parameters as in Fig. 2. 